\title{
Structure and effector-binding analysis of a PreQ1-III riboswitch suggests a fold that is tolerant to binding pocket mutations.
}

\author{
K Srivastava ${ }^{1}$, J Wedekind ${ }^{2}$, J Jenkins ${ }^{3}$, T Rohe ${ }^{4}$ \\ ${ }^{1}$ Department of Biophysics and Biochemistry, University of Rochester Medical Center, Rochester, \\ NY, ${ }^{2}$ Dept of Biochemistry \& Biophysics, University of Rochester, ${ }^{3}$ University of Rochester, \\ ${ }^{4}$ University of Rochester, ROCHESTER, NY \\ KumariYoshita_Srivastava@URMC.rochester.edu
}

Riboswitches are metabolite-sensing RNA motifs present mostly in the 5'-leader sequences of bacterial mRNAs, where they control gene regulation. The class-III preQ1 riboswitch is found in bacteria of the Ruminococcaceae family, which compose the human gut microbiome. I am working with the class III riboswitch from Faecalibacterium prausnitzii (Fpr), an anti-inflammatory commensal bacterium whose deficiency has been associated with Crohn's disease. Although the global fold of the preQ1-III riboswitch is unique, ten nucleotide bases that compose the effector binding pocket are structurally identical to those of the class II riboswitch found in Streptococcus pneumoniae (Spn) - the leading cause of bacterial meningitis in adults. Given recent efforts to develop inhibitors that target riboswitches, it has become important to investigate the fundamental molecular mechanisms of riboswitches that bind the same effector but function in commensal versus pathogenic settings, such as the class III and class II preQ1 riboswitches.

A co-crystal structure of the Fpr preQ1-III riboswitch determined by our lab revealed a chemical interaction network that links the effector-binding pocket to the distal Shine-Dalgarno sequence, which functions in downstream gene regulation. We have prepared several mutants, spatially identical mutations in a preQ1-II riboswitch, including A84G, A52G, $\Delta 84$, and U8C/A85G to assess whether identical binding pocket mutations are affected by different riboswitch folds. PreQ1 binding analysis by isothermal titration calorimetry (ITC) revealed that the equivalent mutations were better tolerated by the class III riboswitch compared to the class II riboswitch. Moreover, our structures of the A84G (PDB ID: 6XKO) and A52G (PDB ID: 6XKN) mutants reveal that the mutant structures are not significantly different from the published wildtype structure, in accord with ITC experiments. We have prepared crystals of $\Delta 84$ and U8C/A85G mutants as well.

Acta Cryst. (2020). A76, a216 